\title{
Role of Hematological Score in Early Diagnosis of Neonatal Sepsis
}

\author{
Dipika Dey ${ }^{1 *}$ \\ Razia Sulltana ${ }^{2}$ \\ Wazir Ahmed ${ }^{3}$ \\ Mahmood Ahmed Chowdhury ${ }^{4}$ \\ Farhana Akter ${ }^{1}$ \\ Srabony Dasgupta ${ }^{1}$
}

\footnotetext{
'Department of Neonatology Chattogram Maa-O-Shishu Hospital Medical College Chattogram, Bangladesh.

${ }^{2}$ Department of Pathology Chattogram Maa-O-Shishu Hospital Medical College Chattogram, Bangladesh.

${ }^{3}$ Head of Institute of Child Health

Chattogram Maa-O-Shishu General Hospital Medical College Chattogram, Bangladesh.

${ }^{4}$ Department of Paediatrics

Chattogram Maa-O-Shishu Hospital Medical College Chattogram, Bangladesh.
}

*Correspondence to:

\section{Dr. Dipika Dey}

Assistant Professor

Department of Neonatology

Chattogram Maa-O-Shishu Hospital Medical College Chattogram, Bangladesh.

Mobile : +88017113928 80

Email: dipikadey880@yahoo.com

$\begin{array}{lll}\text { Date of Submission } & : & 14.06 .2019 \\ \text { Date of Acceptance } & : & 30.06 .2019\end{array}$

www.banglajol.info/index.php/CMOSHMCJ

\begin{abstract}
Background: Neonatal sepsis is one of the major causes of morbidity \& mortality in the newborn, more so in the developing countries.

Objective: The objective of this study was to evaluate the applicability of Haematological Scoring System (HSS) in early diagnosis of sepsis and its compatibility with C-reactive protein (CRP) and blood culture.

Materials and methods: This prospective study consisted of 205 neonates admitted at neonatal intensive care unit of Chattogram Maa-shishu-o General Hospital with clinical suspicion of neonatal sepsis, from July 2017 to December 2017. The neonatal hematological parameter was measured in all cases. Blood culture and CRP estimation were also performed. Blood culture is considered as gold standard for diagnosis of sepsis. Score 3 and more was considered as positive.

Results: Out of 205 neonates of our study population, forty one (20\%) had proven sepsis according to culture. Total leukocyte count showed high sensitivity \& least specificity, immature to mature neutrophil ratio showed high sensitivity and high specificity. Platelet count showed high negative predictive value and least positive predictive value. The HSS was found to have a sensitivity of $82.9 \%$, specificity of $79.8 \%$, positive predictive value was $50.74 \%$ and negative predictive value was $94.92 \%$. Considering the high sensitivity and negative predictive value, this study implies that score $\geq 3$ were more reliable as a screening tool for sepsis than any of the individual hematological parameter.

Conclusion: HSS is a simple, easy and rapid adjunct for the diagnosis of clinically suspected cases of neonatal sepsis. It also provides an effective guideline to make decisions regarding judicious use of antibiotic therapy.
\end{abstract}

Key words : Neonatal sepsis; Blood culture; Hematological scoring system.

\section{INTRODUCTION}

Neonatal sepsis is a clinical syndrome resulting from pathophysiologic effects of local \& systemic infection in the 1 st month of life. Septicemia refers to bacteremia along with signs and symptoms resulting from the microorganisms or their toxic products in the blood ${ }^{1}$. Sepsis has been reported in upto $25 \%$ children in intensive care units ${ }^{2}$. About 4 million deaths occur worldwide annually from neonatal sepsis and $99 \%$ of them occur in developing countries ${ }^{3}$. Sepsis not only causes mortality but also causes some serious morbidities such as seizures, neuro-developmental disorders, sensory neural hearing loss, ocular disturbances etc ${ }^{4}$.

The newborns can acquire the infection from mother through transplacental routes, ascending infection, during passage through an infected birth canal or exposure to infected blood at delivery ${ }^{5}$. In comparison to older children or adults, newborns have weaker immune system, therefore are more susceptible to bacterial invasion, premature babies being even more vulnerable ${ }^{6}$. 
With early diagnosis, it is possible to treat sepsis and prevent its life-threating complications. But the early clinical features of sepsis are often non-specific that makes it difficult for the physician to come to a definite diagnosis ${ }^{7}$. Therefore, often empirical use of antibiotics are seen with a little doubt of sepsis. Though earliest administration of antibiotics is effective in combating acute infections, it also increases the emergence of antibiotic resistant organisms which is a great concern nowa-days ${ }^{7,8}$.

The gold standard for diagnosis of sepsis is blood culture, but the technique is time consuming and requires an well- equipped laboratory, which is not available in most of the hospitals of developing countries. A disadvantage of culture based diagnosis is the assay time of up to $48-72$ hours, yields a positive result in $10-60 \%$ of causes ${ }^{9}$.

Various studies have been done regarding the early diagnosis of neonatal sepsis, which have shown that hematological parameters are simple, quick and cost-effective tools for this purpose $^{10}$. Both sensitivity and specificity are increased when the hematological parameters were studied together as a combination of tests. By early prediction of sepsis, these parameters help to start early treatment using appropriate antibiotics.

A diagnostic test is considered ideal, when it is quick, costeffective, having high sensitivity, specificity, positive and negative predictive value. Considering above criteria Hematological Scoring System of Rodwell is reliable for early diagnosis of neonatal sepsis as it includes all parameters, and shown to be significantly associated with sepsis ${ }^{10}$.

Hematological Scoring System (HSS) that we studied includes Total leucocyte count, Absolute neutrophil count, Immature to Total neutrophil ratio (I:T). Degenerative change in PMN (eg: toxic granulation, vacuolation), Platelet count. Measurement of CRP and blood culture was also done.This study was done to evaluate the utility of the HSS in the early diagnosis of neonatal sepsis.

\section{MATERIALS AND METHODS}

It was a prospective cross sectional study which was done at the Neonatology Department of Chattogram Maa-Shishu-O General Hospital, Chattogram from July 2017 to December 2017 in co-ordination with Pathology Department. Following were the inclusion criteria: Maternal history of prolong rupture of membrane, maternal foul smelling vaginal discharge, intrapartum fever, maternal urinary tract infection or clinical feature of sepsis, such as, reluctance to feed, poor activity, fever $\geq 101^{\circ}$ F. All the neonates with perinatal asphyxia, history of passage of meconium in utero, gross congenital anomaly, any prior administration of antibiotic, history of sibling death were excluded from the study. The study population were scored as per as the Hematological scoring system. Blood Culture and CRP estimation were also done. Score of more than 3 was considered as positive (Table I).
Table I : Hematological scoring system

$\begin{array}{llc}\text { Criteria } & \text { Abnormality } & \text { Score } \\ \text { Total Leucocyte Count (Cells/cumm) } & <5,000 \text { or }>20,000 & 1 \\ \text { Absolute Neutrophil Count (Cells/cumm) } & <1,800 \text { or } \geq 5,400 & 1 \\ \text { IT Ratio } & \geq 0.2 & 1 \\ \text { Degenerative Changes In Neutrophil } & \text { Toxic-granules, } & \\ & \text { cytoplasmic vacuoles } & 1 \\ \text { Platelet Count (Cells/cumm) } & <1,50,000 & 1\end{array}$

Findings of HSS were recorded in a proforma and later compared with results of CRP and Blood Cultures.

\section{RESULTS}

Table II : Presentation profile of study cohort

\begin{tabular}{|c|c|c|}
\hline Variable & Frequency & Percentage \\
\hline \multicolumn{3}{|l|}{ Age } \\
\hline - $0-7$ & 121 & 59 \\
\hline - $8-14$ & 19 & 9.26 \\
\hline - $15-21$ & 45 & 21.9 \\
\hline$\bullet \geq 21$ & 20 & 9.7 \\
\hline \multicolumn{3}{|l|}{ Gender } \\
\hline - Male & 134 & 65 \\
\hline - Female & 71 & 35 \\
\hline \multicolumn{3}{|l|}{ Place of birth } \\
\hline - Home & 54 & 26.3 \\
\hline - Hospital & 151 & 73.6 \\
\hline \multicolumn{3}{|l|}{ Gestation } \\
\hline - Term & 146 & 71.2 \\
\hline - Preterm & 59 & 28.78 \\
\hline \multicolumn{3}{|l|}{ Mode of delivery } \\
\hline - Vaginal & 126 & 61.4 \\
\hline - Cesarean section & 79 & 38.5 \\
\hline \multicolumn{3}{|l|}{ Birth weight } \\
\hline$\bullet 1.5$ & 9 & 4.3 \\
\hline $1.5-2.5$ & 62 & 30.24 \\
\hline$\cdot 2.5$ & 134 & 65.36 \\
\hline \multicolumn{3}{|l|}{ Risk factors } \\
\hline - PROM & 56 & 27.3 \\
\hline - Maternal fever & 38 & 18.5 \\
\hline - Poor Neonatal feeding & 159 & 77.5 \\
\hline - Depressed neonatal reflexes & 136 & 66.3 \\
\hline
\end{tabular}

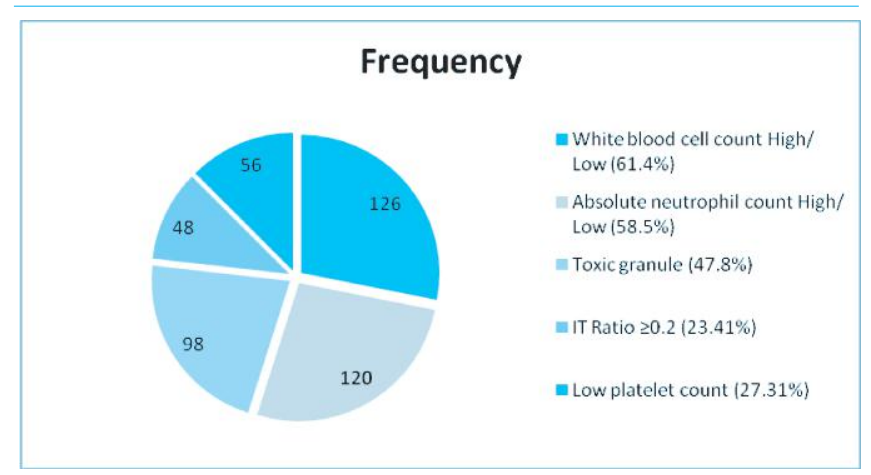

Figure 1 : Distribution of Neonates according to the hematological parameters 
Table III : Comparison of HSS with CRP

\begin{tabular}{lrrr} 
& \multicolumn{2}{c}{ CRP } & $\begin{array}{r}\text { Correct } \\
\text { Positive }\end{array}$ \\
& Negative & Total \\
Sepsis on HSS Positive & 44 & 23 & 67 \\
Negative & 8 & 130 & 138 \\
Total & 52 & 153 & 205 \\
\hline
\end{tabular}

Sensitivity $=84 \% \quad$ Specificity $=85 \%$

Table IV : Comparison of HSS with blood culture

\begin{tabular}{lccr}
\multicolumn{2}{c}{ SEPSIS ON BLO0D CULTURE } & Correct \\
& Positive & Negative & Total \\
Sepsis on HSS Positive & 34 & 33 & 67 \\
Negative & 7 & 131 & 138 \\
Total & 41 & 164 & 205 \\
\hline
\end{tabular}

Sensitivity $=82.9 \%, \quad$ Specificity $=79.8 \%$

Table V : Distribution of hematological score by blood culture \& sensitivity

\begin{tabular}{lrrr}
$\begin{array}{lrrr}\text { Blood CS } \\
\text { Score }\end{array}$ & $\begin{array}{r}\text { Positive } \\
\text { Number(\%) }\end{array}$ & $\begin{array}{r}\text { Negative } \\
\text { Number(\%) }\end{array}$ & P value \\
Score 1 & $3(7.3 \%)$ & $71(43.2 \%)$ & 0.017 \\
Score 2 & $4(9.75 \%)$ & $60(36.58 \%)$ & 0.594 \\
Score 3 & $14(34.1 \%)$ & $20(12.10 \%)$ & 0.000 \\
Score 4 & $20(48.78 \%)$ & $13(7.92 \%)$ & 0.000 \\
\hline
\end{tabular}

*Chi square test was done to measure the level of significance. Figure within parentheses indicates in percentage.

Table VI : Comparison between hematological parameters and blood culture

\begin{tabular}{lccllll} 
Blood CS & & & & & & \\
& Positive & Negative & Sensitivity & Specificity & PPV & NPV \\
\hline \multicolumn{2}{l}{ Leucocytosis/ Leucopenia 40} & 85 & $97.56 \%$ & $47.85 \%$ & $32 \%$ & $98.73 \%$ \\
Thrombocytopenia & 39 & 17 & $95.12 \%$ & $89.63 \%$ & $69.64 \%$ & $98.65 \%$ \\
IT Ratio $(\geq 0.2)$ & 40 & 8 & $97.56 \%$ & $95.12 \%$ & $83.33 \%$ & $99.36 \%$ \\
ANC (High/Low) & 40 & 80 & $97.56 \%$ & $51.21 \%$ & $33.33 \%$ & $98.8 \%$ \\
Toxic Granules $(+)$ & 38 & 60 & $92.68 \%$ & $63.41 \%$ & $38.77 \%$ & $97.19 \%$ \\
\hline
\end{tabular}

\section{DISCUSSION}

Neonatal sepsis due to its high mortality rate, still a diagnostic and treatment challenge for the neonatal health care providers. Early diagnosis helps the clinician in instituting antibiotic therapy at the earliest, which in turn reduces the mortality rates in the neonates.

205 newborns were included in this study. Among them 20\% (41) newborns were diagnosed as proven sepsis by blood culture. However suspected sepsis groups 32.68\% (67) comprises a difficult diagnostic group \& count not be ignored as negative blood culture does not rule out fatal infection ${ }^{11}$.

Among the infected newborns, male (65\%) were found predominant. It may be due to the factors regulating the synthesis of a globulin are situated on the X-Chromosome. Males have only one X-Chromosome, that's why less immunologically protected than the females. Premature Rupture of Membrane (PROM) is an important risk factor for neonatal sepsis as it poses of ascending infection to the fetus and it was found in $27.3 \%$ cases in our study.

In this present study, sensitivity of Total Leukocyte Count (TLC) was found to be $97.56 \%$, specificity $47.85 \%$ with PPV $32 \%$ and NPV $98.73 \%$. This result was consistent with other studies $^{12}$. So it can be said that total leucocyte count acts as a good parameter for confirmation of sepsis.

Ghosh et al. and Narasimha et al. reported that Immature: Total (I:T) PMN (Polymorphoneuclear Neutrophil) ratio acts as reliable indicator of neonatal sepsis ${ }^{13,14}$. In this study, IT ratio $\geq$ 0.2 had a sensitivity, specificity, PPV and NPV of $07.56 \%$, $95.12 \%, 83.33 \%$ and $99.36 \%$ respectively which was consistent with other reports ${ }^{13,14}$. The production of unusal PMNs during infection and stress induced leukopoesis is reflected in blood picture as presence of toxic granules. Although the count is not always increased, their presence invariably indicates sepsis. Thrombocytopenia in neonatal sepsis occurs due to Disseminated Intravascular Coagulation (DIC) and the damaging effects of endotoxin on platelets. In this study thrombocytopenia was found in $27.31 \%$ cases with sensitivity of $95.12 \%$, specificity of $89.63 \%$, PPV of $69.64 \%$ and NPV of $98.65 \%$. This parameter could be used as an early but nonspecific marker for sepsis. These results were consistent with other study ${ }^{15}$.

The mortality and morbidity associated with sepsis is high, that's why tests with high sensitivity and Negative Predictive Value (NPV) are most desirable because all infants with sepsis have to be indentified ${ }^{16}$. HSS is a combination of different parameters, which makes it a better diagnosis tool, because no individual parameter was found more accurate than another.

Although the chance of sepsis is more with higher score on HSS, it is still a necessity to simplify \& standardize this test. Globally different rapid diagnostic test such as automated blood culture system, DNA probes, flurometric detection system are available for detection of micro-organisms, but still HSS may be considered as a good screening test for early differentiation of the septicemic groups from the non-septicemic ones. Moreover, HSS has high sensitivity \& specificity with increasing score increasing certainty of sepsis ${ }^{17}$.

\section{CONCLUSION}

HSS in an effective tool for early diagnosis of neonatal sepsis which makes it possible to start antibiotic rationally and prevent irrational use of antibiotic, thereby minimizes the emergence of resistant organism. It is also a simple, cost-effective tool that can be done at the primary health care center as a routine screening procedure.

\section{LIMITATIONS}

- Single center study

- Short duration of the study.

\section{DISCLOSURE}

All the authors declared no competing interest. 


\section{REFERENCES}

1. $\quad$ Robertson NR. Text Book of Neonatology by Robertson. $2^{\text {nd }}$ ed. Philadelphia: Churchill Livingstone Publishers. 1992; 925-999.

2. Wynnj, Cornell TT, Wong HR, Shanley TP. The Host Response to Sepsis and Developmental Impact. Pediatrics. 2010;125:1031-1041.

3. Darmstadt Gl, SahaSk, Choi Y, Arifeen SE. Population-based incidence and etiology of community acquired neonatal bacteremia in Mirzapur, Bangladesh:Anobserrational study. J Infect dis. 2009; 200: 906-915.

4. Stoll BJ. Infections of the Neonatal Infant. In: Kliegman RM, Behrman RE, Jenson HB, Stanton BF, editors. Nelson Textbook of Pediatrics. $18^{\text {th }}$ ed. Saunders. 2007; $794-809$

5. Behrman RE, Kliegman RM, Jeanson H, Nelson Textbook of Pediatrics. $17^{\text {th }}$ ed. Philadelphia: WB. Saunders. 2004;811-814.

6. Sankar MJ, Agarwal R, Deorari AK, Paul. Sepsis in the newborn. Indian J Pediatr. 2008;75: 261-266.

7. Shirazi H, Riaz S, Tahir R. Role of the Hematological Profile in Early Diagnosis of Neonatal sepsis. Ann Pak Inst Med Sci. 2010; 6(3): 152-156.

8. Edgar JDM, Gabriel V, Gallimore JR, MC Millan SA.A prospective study of the sensitivity, specificity and diagnostic performance of soluble inter cellular adhesion molecule 1, highly sensitive c-reactive protein, soluble E-selection and serum amyloid A in the diagnosis of neonatal infection. BMC Pediatr. 2010;10:22-25.

9. Haque KN. Neonatal sepsis in the very low birth weight preterm Infants: Part 2: review of definition, Diagnosis and Management. J Med Sci. 2010;3(1):11-27.

10. Rodwell RL, Leslie AL, Tudehope DI. Early diagnosis of neonatal sepsis using a Hematologic scoring system. J Pediatric. 1988;112:761-767.

11. Haider S, Riaz S, Tahir R. Role of hematological profile in early diagnosis of neonatal sepsis. Ann Pak inst Med Sci. 2010;6:152-156.

12. Fowlie PW, Schmidt B. Diagnostic tests for Bacterial infection from birth to 90 days- A systemic review. Arch Dis Child Fetal Neonatal. 1998;78:92-98

13. Ghosh S, Mittal M, Jaganathan G. Early diagnosis of neonatal sepsis usinga hematological scoring system. Indian J Med Science. 2001;55:495-500.

14. Narasimha A, Kumar ML. Significance of Hematological Scoring System (HSS) in early diagnosis of neonatal sepsis. Indian J Hematol Blood Transfus. 2011; 27; 14-17.

15. Shirin M, Hossain MM, Mamun MAA, Chowdhury NA, Qadar A. Sensitivity \& Specificity of C-reactive Protein (CRP) and thrombocytopenia in the diagnosis of neonatal sepsis. Bangladesh J child health. 2005; 29 (2):41-45.

16. Zak MEIS. Sayed HEI, Evaluation of microbiologic and hematologic parameters and E-selectin as early predictors for outcome of neonatal sepsis. 2008;2:17-19.

17. Khair KB, Rahman MA, Sultana T, Roy CK, Rahman MQ, Shahidullah M, et al. Role of hematological scoring system in early diagnosis of neonatal septicemia. BSMMU J.2010;3:62-67. 